\title{
Living donor liver transplantation should be cautiously considered as initial treatment in recurrent hepatocellular carcinoma within the Milan criteria after curative liver resection
}

\author{
Jong Man Kim ${ }^{1}$, Jae-Won Joh ${ }^{1}$, Nam-Joon $\mathbf{Y i}^{2}$, Gyu-Seong Choi ${ }^{1}$, Choon Hyuck David Kwon ${ }^{3}$, \\ Kwang-Woong Lee ${ }^{2}$, Kyung-Suk Suh ${ }^{2}$
}

${ }^{1}$ Department of Surgery, Samsung Medical Center, Sungkyunkwan University School of Medicine, Seoul, Republic of Korea; ${ }^{2}$ Division of HBP Surgery, Department of Surgery, Seoul National University College of Medicine, Seoul, Republic of Korea; ${ }^{3}$ Department of Surgery, Digestive Disease and Surgery Institute, Cleveland Clinic, Cleveland, Ohio, USA

Contributions: (I) Conception and design: JM Kim, JW Joh, NJ Yi; (II) Administrative support: JM Kim; (III) Provision of study materials or patients: JM Kim; (IV) Collection and assembly of data: JM Kim, GS Choi, CH Kwon, KW Lee, KS Suh; (V) Data analysis and interpretation: All authors; (VI) Manuscript writing: All authors; (VII) Final approval of manuscript: All authors.

Correspondence to: Jae-Won Joh, MD, PhD. Professor, Department of Surgery, Samsung Medical Center, Sungkyunkwan University School of Medicine, Irwon-Ro 81, Gangnam-Gu, Seoul 06351, Republic of Korea. Email: jw.joh@samsung.com.

Background: Insufficient data are available about patient survival following different treatments for recurrent hepatocellular carcinoma (HCC) after primary hepatectomy. We retrospectively investigated the effectiveness of various treatment methods.

Methods: From 2005 to 2011, 515 hepatectomy patients who developed recurrence within the Milan criteria (MC) were grouped by treatment modality into living donor liver transplantation (LDLT), hepatic re-resection (RR), radiofrequency ablation (RFA), transarterial chemoembolization (TACE), percutaneous ethanol injection (PEI), and combination of TACE and RFA (TACE-RFA) group.

Results: Disease-free survival and patient survival after first HCC recurrence were compared according to treatment strategies. TACE ( $\mathrm{n}=230,44.7 \%)$, RFA ( $\mathrm{n}=171,33.2 \%)$, PEI ( $\mathrm{n}=35,6.8 \%)$, RR ( $\mathrm{n}=45,8.7 \%)$, salvage LDLT $(n=21,4.1 \%)$, and TACE-RFA $(n=13,2.5 \%)$ were all used as the first treatment in recurrent HCC within the MC. The disease-free survival curve from $1^{\text {st }} \mathrm{HCC}$ recurrence in the PEI group was lower than in the other groups $(\mathrm{P}=0.004)$. The RR, salvage LDLT, and TACE-RFA groups showed good longterm prognosis. The patient survival rate at 3 years after $1^{\text {st }} \mathrm{HCC}$ recurrence was $45.2 \%$ in TACE, $51.7 \%$ in RFA, 39.8\% in PEI, 38.2\% in RR, $81.4 \%$ in salvage LDLT, and $80.8 \%$ in the TACE-RFA group. Thus, the patient survival curve in the salvage LDLT and TACE-RFA groups was higher than in the other groups $(\mathrm{P}<0.001)$.

Conclusions: Long-term outcomes for patients with recurrent $\mathrm{HCC}$ within the MC do not differ with the first treatment strategies, except for PEI. Salvage LDLT does not prevent HCC recurrence, but it increases patient survival compared with the other treatment strategies.

Keywords: Hepatocellular carcinoma (HCC); Milan criteria (MC); surgery; transplantation; living donors; outcome

Submitted Nov 30, 2019. Accepted for publication Feb 07, 2020.

doi: $10.21037 /$ atm.2020.02.170

View this article at: http://dx.doi.org/10.21037/atm.2020.02.170 


\section{Introduction}

Hepatic surgical resection (SR) is considered as curative treatment in hepatocellular carcinoma (HCC) in the patients with preserved liver function (1). However, HCC recurrence is more than $50 \%$ within 5 years after surgery, which is the biggest problem after SR $(2,3)$. Liver transplantation (LT) is the most effective treatment for HCC based on the Milan criteria (MC) (4). In Eastern Asia, SR is a priority for HCC patients with sufficient liver function due to limitation of deceased donation, whereas LT is performed as the initial treatment in Western countries.

Intrahepatic recurrence after SR is very common. Various treatments, such as LT, SR, radiofrequency ablation (RFA), transarterial chemoembolization (TACE), percutaneous ethanol injection (PEI), and combination of TACE and RFA (TACE-RFA), are being applied to these patients, and the response to treatment influences long-term outcomes (5).

Salvage liver transplantation (SLT) is an ideal treatment for patients with intrahepatic HCC recurrence or decompensated liver function after SR (6). SLT is expected to have a good prognosis in patients with intrahepatic recurrence of within MC (7). However, the lack of deceased donors makes it difficult for HCC patients to undergo LT. Therefore, SLT is a very attractive treatment when intrahepatic recurrence occurs after SR as initial treatment $(6,7)$. These treatment strategies are readily available and show similar outcomes when compared to primary LT (8). However, various locoregional treatments are used because SLT after SR is not easy. Therefore, optimal retreatment strategies or consensus treatment strategies regarding intrahepatic recurrent HCC have not been clearly established.

The aim of this study was to compare the effects of various treatments on patients with intrahepatic HCC recurrence with MC after SR in solitary HCC patients.

\section{Methods}

\section{Patients}

This study included patients who underwent SR for treatment-naïve single HCC, which was diagnosed by preoperative radiologic images, in Seoul National University Hospital (SNUH) (n=938) and Samsung Medical Center (SMC) $(\mathrm{n}=1,382)$ from January 2005 to December 2011. HCC was diagnosed on the basis of several guidelines $(9,10)$ and histologically confirmed after SR. We included patients with intrahepatic HCC recurrence within the MC after SR in two centers ( $\mathrm{n}=515)$ (Figure 1), and present study was approved by Institutional Review Board of two centers.

Inclusion criteria included curative SR in treatment-naïve single HCC patients with Child-Pugh class A. Ruptured HCC, mixed HCC and cholangiocarcinoma, R1 resection, non-Korean, age $\leq 18$ years, history of malignancy other than HCC, patients received adjuvant therapy after SR, loss to follow-up after hepatectomy were excluded. Patient medical information was retrospectively investigated.

\section{Surgery and surveillance}

Surgical procedure during SR and histological evaluations have been described previously $(2,3)$. All patients who underwent SR visited outpatients 1 month after the surgery and later evaluated liver function tests, tumor markers, and radiological evaluation every 2 to 3 months. Radiologic evaluations included radiologic evaluation was performed by alternating liver computed tomography (CT) and liver magnetic resonance imaging (MRI) every 3 months for 2 years after surgery.

\section{Treatment of recurrence}

When a recurrence was detected during follow-up after primary curative SR in solitary HCC patients, patients were hospitalized and treated within 1 month after diagnosis. RR was performed in oligonodular HCC patients with ChildPugh class A. RFA or PEI was considered in solitary HCC less than $3 \mathrm{~cm}$ when RR was difficult and dangerous. TACE was considered if serum total bilirubin level was less than $2 \mathrm{mg} / \mathrm{dL}$ without satisfying the previous criteria. Salvage LDLT was considered to have potential living donor and be difficult to use RR, PEI, RFA, and TACE due to decompensated liver function. TACE-RFA was performed in SMC in the case of small solitary HCC less than $3 \mathrm{~cm}$ where RFA was difficult because of tumor location. Target therapy and systemic chemotherapy were not considered as treatment in intrahepatic recurrent HCC patients.

\section{Statistical analysis}

Categorical variables are expressed in number and percentage and continuous variables are described as median and range. Comparisons between the treatment groups (LDLT, RR, RFA, PEI, TACE, and TACE-RFA) were performed by the Chi-square test or Fisher's exact test. A one-way analysis of variance (ANOVA) was used 


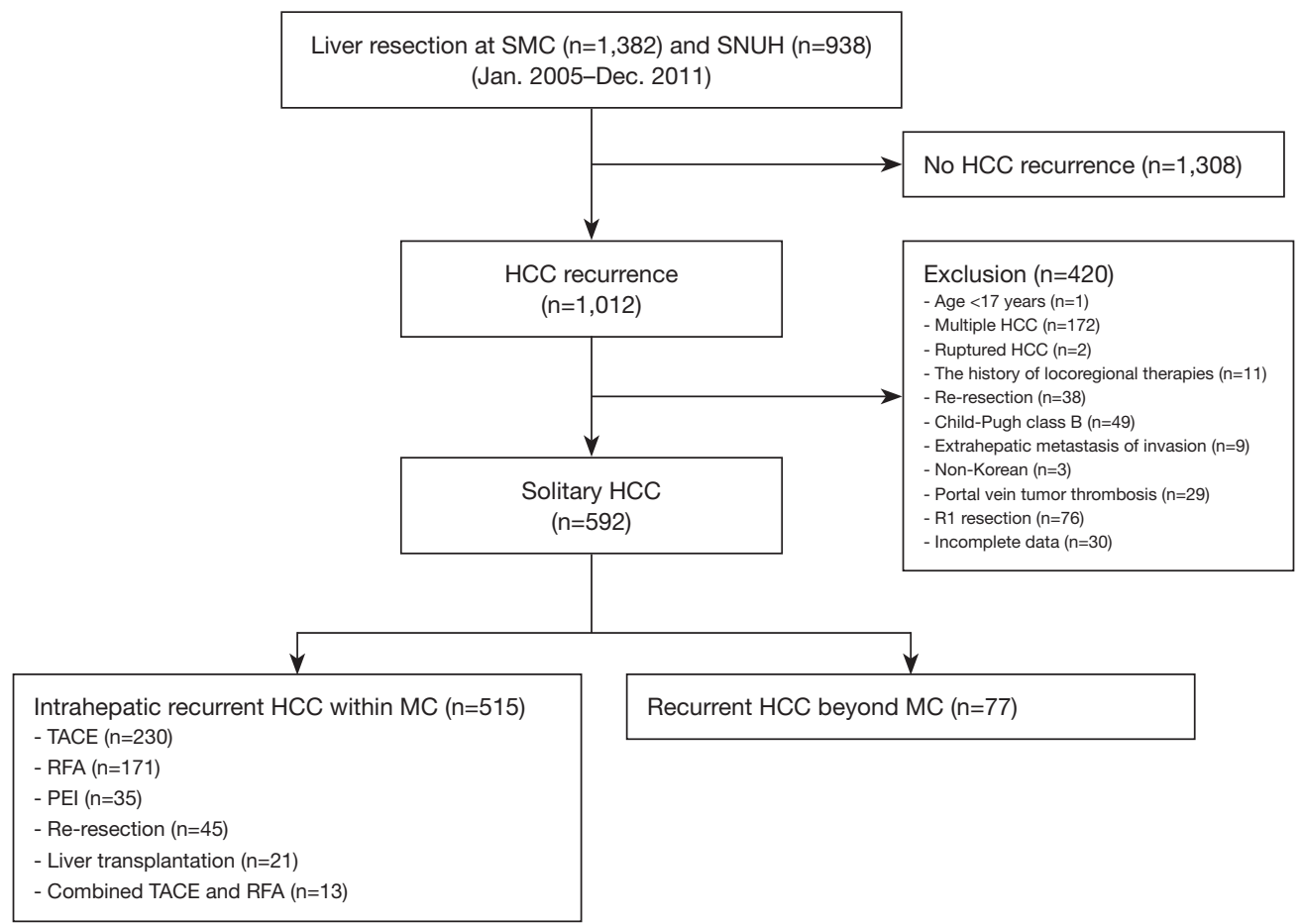

Figure 1 Patient selection process and inclusion/exclusion criteria. SMC, Samsung Medical Center; SNUH, Seoul National University Hospital; HCC, hepatocellular carcinoma; TACE, transarterial chemoembolization; RFA, radiofrequency ablation; PEI, percutaneous ethanol injection; MC, Milan criteria.

for continuous variables. Disease-free survival (DFS) and patient survival (PS) were considered from the date of treatment for recurrent $\mathrm{HCC}$ to a $2^{\text {nd }} \mathrm{HCC}$ recurrence and the last follow-up or death, respectively. Survival rates were analyzed using the Kaplan-Meier method with log-rank test. All tests were two-sided, and statistical significance was defined as $\mathrm{P}<0.05$. All statistical analyses were performed using SPSS ver. 22.0 (SPSS, Inc., Chicago, IL, USA).

\section{Results}

\section{Patient groups and clinicopathologic characteristics}

Recurrent HCC within the MC after curative SR developed in 515 patients. TACE $(n=230,44.7 \%)$, RFA $(n=171$, $33.2 \%)$, PEI ( $\mathrm{n}=35,6.8 \%), \operatorname{RR}(\mathrm{n}=45,8.7 \%)$, LDLT $(\mathrm{n}=21$, $4.1 \%)$, and TACE-RFA ( $n=13,2.5 \%)$ were all used as first treatments in recurrent $\mathrm{HCC}$ within the MC. All recurrent HCC patients were Child-Pugh class A when treated.

Median preoperative platelet counts in the LT and TACE-RFA groups were lower than in the TACE, RFA, and PEI groups $(\mathrm{P}=0.02)$. In addition, serum albumin levels differed significantly. However, gender, age, etiology, body mass index, hemoglobin levels, total bilirubin, aspartate transaminase (AST), alanine transaminase (ALT), international normalized ratio (INR), creatinine, indocyanine green retention rate at 15 minutes (ICG-R15), alpha-fetoprotein (AFP), and the proportion of preoperative within MC did not differ (Table 1).

The proportion of grade 3 or 4 in the LDLT group was $38.1 \%$, but the proportion of grade 3 or 4 in the other groups was $7.7 \%$ to $28.6 \%(\mathrm{P}=0.02)$. The incidence of intrahepatic metastasis in the TACE group was $17.8 \%$, but the incidence in the other groups was $0 \%$ to $7.3 \%(\mathrm{P}=0.002)$. The frequency of major liver resection, tumor size, the presence of tumor necrosis or hemorrhage, microvascular invasion, and portal vein tumor thrombosis (PVTT) did not differ among groups (Table 2).

\section{HCC recurrence}

Median DFS in the RR and TACE-RFA groups was 22 months (range, 2-63 months) and 23 months (range, 9-75 months). Median DFS in the other groups was 13 to 


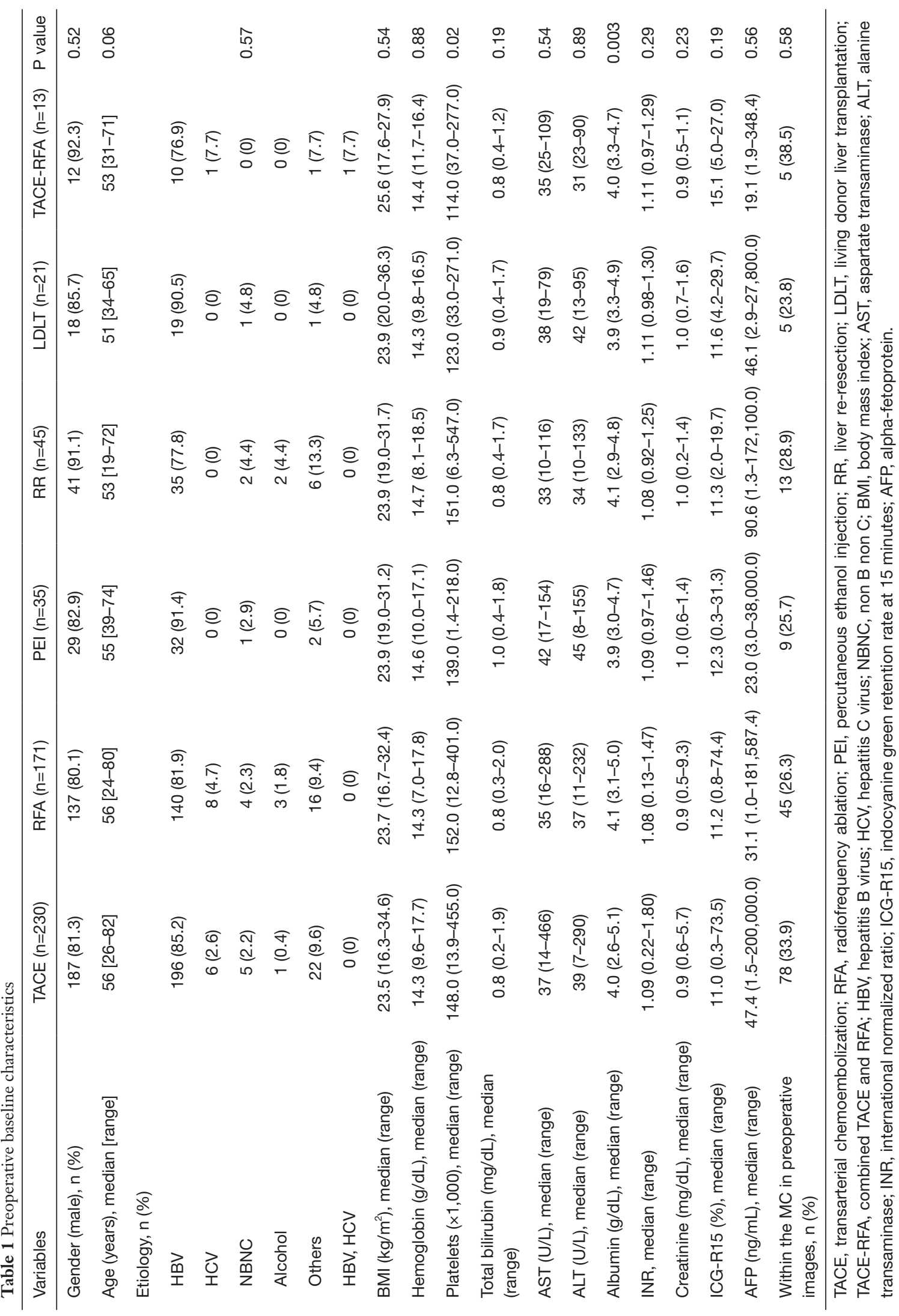


Table 2 Perioperative and pathologic characteristics

\begin{tabular}{|c|c|c|c|c|c|c|c|}
\hline Variables & TACE $(n=230)$ & RFA $(n=171)$ & PEI $(n=35)$ & $\mathrm{RR}(\mathrm{n}=45)$ & $\operatorname{LDLT}(n=21)$ & TACE-RFA ( $n=13)$ & $P$ value \\
\hline Tumor size $(\mathrm{cm})$, median (range) & $3.8(1.0-18.0)$ & $3.5(1.0-15.9)$ & $3.2(1.2-11.0)$ & $3.6(1.0-21.0)$ & $3.6(1.2-16.5)$ & $3.1(1.0-17.0)$ & 0.21 \\
\hline Grade 3 or $4, \mathrm{n}(\%)$ & $38(16.5)$ & $22(12.9)$ & $10(28.6)$ & $10(22.2)$ & $8(38.1)$ & $1(7.7)$ & 0.02 \\
\hline Tumor hemorrhage, n (\%) & $94(40.9)$ & $74(43.3)$ & $10(28.6)$ & $17(37.8)$ & 7 (33.3) & $7(53.8)$ & 0.89 \\
\hline Microvascular invasion, $\mathrm{n}(\%)$ & $111(48.3)$ & $70(40.9)$ & $15(42.9)$ & $17(37.8)$ & 7 (33.3) & $5(38.5)$ & 0.52 \\
\hline PVTT, n (\%) & $8(3.5)$ & $4(2.3)$ & $3(8.6)$ & $0(0)$ & $2(9.5)$ & $0(0)$ & 0.15 \\
\hline Intrahepatic metastasis, n (\%) & $34(14.8)$ & $11(6.4)$ & $1(2.9)$ & $1(2.2)$ & $0(0)$ & $0(0)$ & 0.002 \\
\hline
\end{tabular}

TACE, transarterial chemoembolization; RFA, radiofrequency ablation; PEl, percutaneous ethanol injection; RR, liver re-resection; LDLT, living donor liver transplantation; TACE-RFA, combined TACE and RFA; PVTT, portal vein tumor thrombosis.

18 months $(\mathrm{P}=0.048)$. Accordingly, the proportion of $\mathrm{HCC}$ recurrence more than 1 year after liver resection in the RR, LDLT, and TACE-RFA groups was more than $70 \%$, whereas in the other groups, it was $53 \%$ to $66 \%(\mathrm{P}=0.02)$. Nearly $99 \%$ of patients showed one tumor when recurrent HCC was diagnosed. The median AFP at HCC recurrence was $11.3 \mathrm{ng} / \mathrm{mL}$ in the $\mathrm{RR}$ group, but whereas in other groups, it was 4.6 to $7.7 \mathrm{ng} / \mathrm{mL}(\mathrm{P}=0.01)$. The median follow-up duration after curative LR did not differ among groups (Table 3).

\section{Comparison by treatment modalities on post-recurrence survival}

The DFS rate at 3 years from $1^{\text {st }} \mathrm{HCC}$ recurrence was $42.7 \%$ in TACE, $48.3 \%$ in RFA, $30.2 \%$ in PEI, $58.1 \%$ in RR, $54.0 \%$ in LDLT, and $56.3 \%$ in the TACE-RFA group. The DFS curve from $1^{\text {st }} \mathrm{HCC}$ recurrence in the PEI group was lower than in the other groups $(\mathrm{P}=0.004)$ (Figure 2). The RR, LDLT, and TACE-RFA groups showed good long-term prognosis.

The PS rate at 3 years after $1^{\text {st }} \mathrm{HCC}$ recurrence was $45.2 \%$ in TACE, $51.7 \%$ in RFA, $39.8 \%$ in PEI, $60.2 \%$ in RR, $81.4 \%$ in LDLT, and $80.8 \%$ in the TACE-RFA group. The PS curve in the LDLT and TACE-RFA groups was higher than in the other groups $(\mathrm{P}<0.001)$ (Figure 2).

\section{Discussion}

The long-term prognosis in recurrent HCC patients after curative LR is unsatisfactory because tumor recurrence is related with patient death. The reported independent prognostic factors for post-recurrence survival are primary tumor status such as satellite nodules or PVTT, and advanced liver cirrhosis in background liver. The time to recurrence and the tumor size, number, and location of recurrence affect PS $(2,3)$. In addition, early diagnosis and treatment of recurrence after liver cancer surgery have a great influence on the prognosis of patients with hepatectomy. The choice of treatment depends on the location of the tumor, performance of patient, and liver reserve function. However, consensus treatment strategies for recurrent intrahepatic HCC have not been established. According to the treatment algorithm in multiple guidelines, reallocating patients with recurrent HCC to treatment as if they were treatment-naïve patients might be inappropriate (10).

All the recurrent HCC patients after curative SR in the present study were Child-Pugh class A and within the MC because we wanted to evaluate the outcomes from different first-line treatments for intrahepatic recurrent HCC with preserved liver function. In this study, a $2^{\text {nd }}$ HCC recurrence in recurrent HCC within the MC did not differ by treatment modality except for the PEI group. PS in the LDLT and TACE-RFA groups was higher than in the other groups. We thought it would show a good prognosis after LDLT as aggressive treatment for recurrent HCC. However, LDLT is not overwhelmingly superior to other therapies in our study.

Previous several studies have reported that aggressive treatments, such as RR and SLT, have survival benefits over non-surgical treatments (11-14). The single recurrent 
Table 3 Characteristics at HCC recurrence

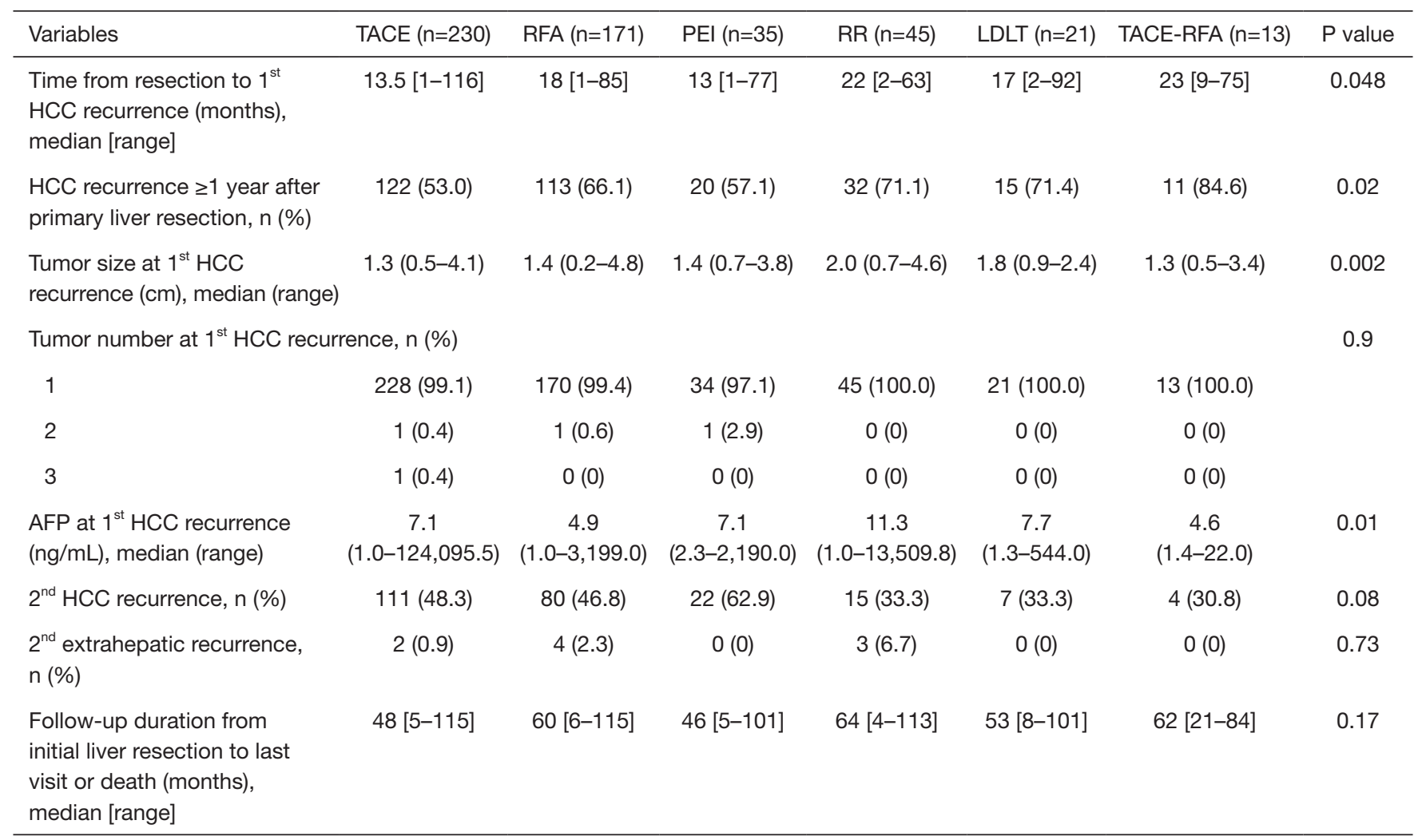

TACE, transarterial chemoembolization; RFA, radiofrequency ablation; PEI, percutaneous ethanol injection; RR, liver re-resection; LDLT, living donor liver transplantation; TACE-RFA, combined TACE and RFA; HCC, hepatocellular carcinoma; AFP, alpha-fetoprotein.
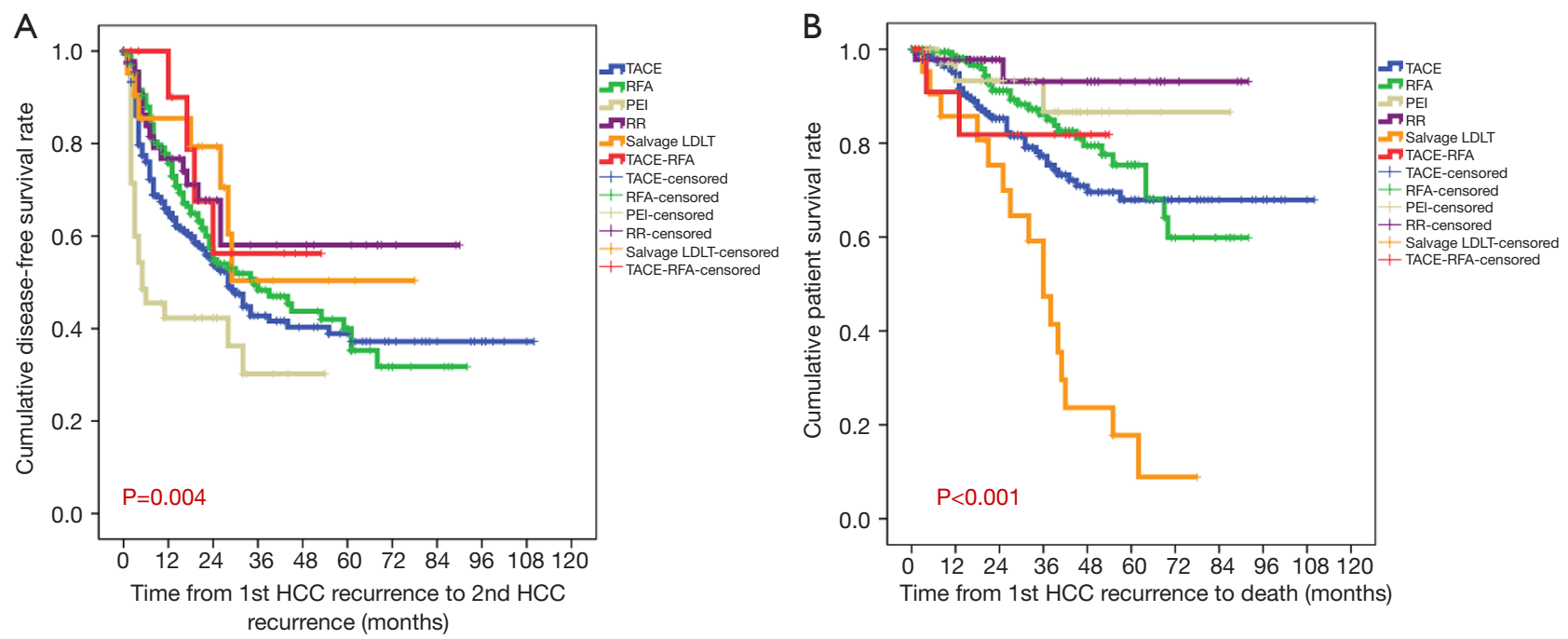

Figure 2 Disease-free survival (A) and patient survival (B) of patients with recurrent HCC within the MC according to treatment strategies. TACE, transarterial chemoembolization; RFA, radiofrequency ablation; PEI, percutaneous ethanol injection; RR, liver re-resection; LDLT, living donor liver transplantation; TACE-RFA, combined TACE and RFA; HCC, hepatocellular carcinoma; MC, Milan criteria. 
HCC at the peripheral site shows a good prognosis after RR or RFA $(15,16)$. Previous propensity score matching study showed that long-term survival in the RFA group was higher than in the RR group (11). In our population, 3-year DFS rate and PS rate from the time of recurrence were $58.1 \%$ and $60.2 \%$ in the RR group and $48.3 \%$ and $51.7 \%$ in the RFA group, respectively. Thus, our study showed similar outcomes between the two groups.

The feasibility of RR or RFA can be limited by multinodular recurrent tumors, difficult tumor locations, a small expected remnant liver volume, poor performance status, medical comorbidities, limited liver functional reserve, and other causes. TACE is an alternative option for those patients. In this study, we found comparable DFS rates and PS rates in the TACE and RFA groups.

Predicting HCC recurrence after SR is associated with tumor aggressiveness. Pathologic reports reflect tumor aggressiveness. The proportion of tumor grade of 3 or 4 in the PEI and LDLT groups was higher than in the TACE, RFA, RR, and TACE-RFA groups. Additionally, the incidence of intrahepatic metastasis in the TACE group was higher than in the other groups. The optimal treatment strategy should ideally be guided by a rigorous risk-versusbenefit assessment, but even such an analysis does not always provide a clear answer.

This study has several limitations. First, our study is retrospective. in our study. Second, the type of treatment was not randomly decided, and treatment selection bias did not completely eliminate in present study. Third, PET/CT or serum inflammatory indices were not evaluated in our study. Fourth, our population contained a higher proportion of patients with hepatitis B virus than reported in Western countries.

In conclusion, our results show that outcomes of patients with recurrent HCC within the MC does not differ with the first treatment strategies, except for PEI. Interestingly, salvage LDLT does not prevent HCC recurrence, but it increases PS compared with the other treatment strategies. However, LDLT as a first treatment strategy in recurrent HCC within the MC after curative resection should be carefully considered because it is a living donor operation.

\section{Acknowledgments}

Funding: None.

\section{Footnote}

Conflicts of Interest: The authors have no conflicts of interest to declare.

Ethical Statement: The authors are accountable for all aspects of the work in ensuring that questions related to the accuracy or integrity of any part of the work are appropriately investigated and resolved. This study was approved by the Samsung Medical Center Institutional Review Board (SMC 2013-05-013) and Seoul National University Hospital Institutional Review Board (H-1303061-474). Written informed consent was exempted from each IRB.

Open Access Statement: This is an Open Access article distributed in accordance with the Creative Commons Attribution-NonCommercial-NoDerivs 4.0 International License (CC BY-NC-ND 4.0), which permits the noncommercial replication and distribution of the article with the strict proviso that no changes or edits are made and the original work is properly cited (including links to both the formal publication through the relevant DOI and the license). See: https://creativecommons.org/licenses/by-nc-nd/4.0/.

\section{References}

1. Cherqui D, Laurent A, Mocellin N, et al. Liver resection for transplantable hepatocellular carcinoma: long-term survival and role of secondary liver transplantation. Ann Surg 2009;250:738-46.

2. Kim JM, Kwon CH, Joh JW, et al. Differences between hepatocellular carcinoma and hepatitis B virus infection in patients with and without cirrhosis. Ann Surg Oncol 2014;21:458-65.

3. Kim JM, Kwon CH, Joh JW, et al. Outcomes after curative hepatectomy in patients with non-B non-C hepatocellular carcinoma and hepatitis B virus hepatocellular carcinoma from non-cirrhotic liver. J Surg Oncol 2014;110:976-81.

4. Mazzaferro V, Regalia E, Doci R, et al. Liver transplantation for the treatment of small hepatocellular carcinomas in patients with cirrhosis. N Engl J Med 1996;334:693-9.

5. Zhang X, Li C, Wen T, et al. Appropriate treatment strategies for intrahepatic recurrence after curative resection of hepatocellular carcinoma initially within the Milan criteria: according to the recurrence pattern. Eur J Gastroenterol Hepatol 2015;27:933-40.

6. Lee S, Hyuck David Kwon C, Man Kim J, et al. Time of hepatocellular carcinoma recurrence after liver resection and alpha-fetoprotein are important prognostic 
factors for salvage liver transplantation. Liver Transpl 2014;20:1057-63.

7. Lee SG. Salvage living-donor liver transplantation to previously hepatectomized hepatocellular carcinoma patients: is it a reasonable strategy? Hepatobiliary Pancreat Dis Int 2013;12:10-1.

8. Hwang S, Lee SG, Moon DB, et al. Salvage living donor liver transplantation after prior liver resection for hepatocellular carcinoma. Liver Transpl 2007;13:741-6.

9. Heimbach JK, Kulik LM, Finn RS, et al. AASLD guidelines for the treatment of hepatocellular carcinoma. Hepatology 2018;67:358-80.

10. Korean Liver Cancer A, National Cancer C. 2018 Korean Liver Cancer Association-National Cancer Center Korea Practice Guidelines for the Management of Hepatocellular Carcinoma. Gut Liver 2019;13:227-99.

11. Song KD, Lim HK, Rhim H, et al. Repeated Hepatic Resection versus Radiofrequency Ablation for Recurrent Hepatocellular Carcinoma after Hepatic Resection: A Propensity Score Matching Study. Radiology

Cite this article as: Kim JM, Joh JW, Yi NJ, Choi GS, Kwon $\mathrm{CH}$, Lee KW, Suh KS. Living donor liver transplantation should be cautiously considered as initial treatment in recurrent hepatocellular carcinoma within the Milan criteria after curative liver resection. Ann Transl Med 2020;8(6):288. doi: 10.21037/ atm.2020.02.170
2015;275:599-608.

12. Meniconi RL, Komatsu S, Perdigao F, et al. Recurrent hepatocellular carcinoma: a Western strategy that emphasizes the impact of pathologic profile of the first resection. Surgery 2015;157:454-62.

13. Ho CM, Lee PH, Shau WY, et al. Survival in patients with recurrent hepatocellular carcinoma after primary hepatectomy: comparative effectiveness of treatment modalities. Surgery 2012;151:700-9.

14. Fuks D, Dokmak S, Paradis V, et al. Benefit of initial resection of hepatocellular carcinoma followed by transplantation in case of recurrence: an intention-to-treat analysis. Hepatology 2012;55:132-40.

15. Faber W, Seehofer D, Neuhaus P, et al. Repeated liver resection for recurrent hepatocellular carcinoma. J Gastroenterol Hepatol 2011;26:1189-94.

16. Umeda Y, Matsuda H, Sadamori H, et al. A prognostic model and treatment strategy for intrahepatic recurrence of hepatocellular carcinoma after curative resection. World J Surg 2011;35:170-7. 